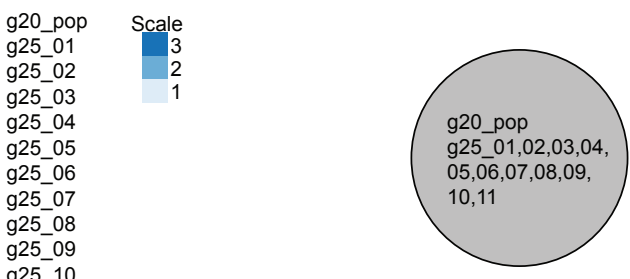

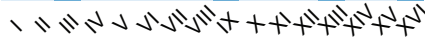

Strain 203

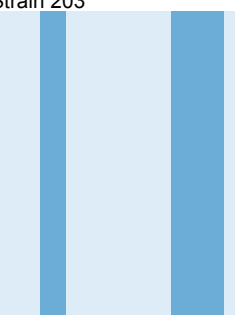

g20_pop

g30 01

g30_02

g30_04

g30_05

g30_06

g30_07

g30_08

g30_09

g30_10

g30_11

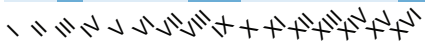

Strain 206

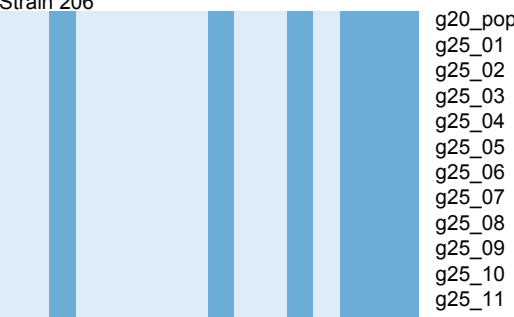

g20 pop

g30_01,02,04,

$05, \overline{06}, 07,08$,

$09,10,11$

0 CIN events

Stable

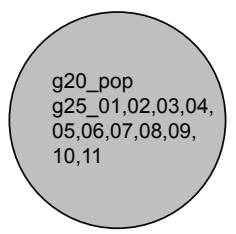

0 CIN events Stable

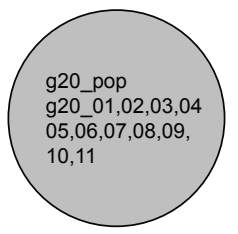

0 CIN events Stable

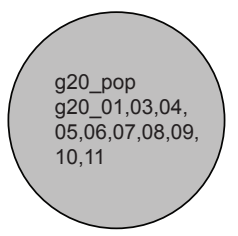

0 CIN events Stable

g20-07

g20_08

g20_09

g20_10

g20_11

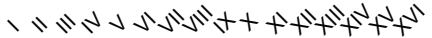

Strain 230

g20_pop

g30_01

g30_02

g30_03

g30_04

g30 05

g30_06

g30 07

g30_08

g30_09

g30 10

g30_11

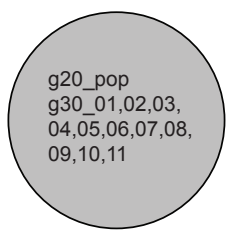



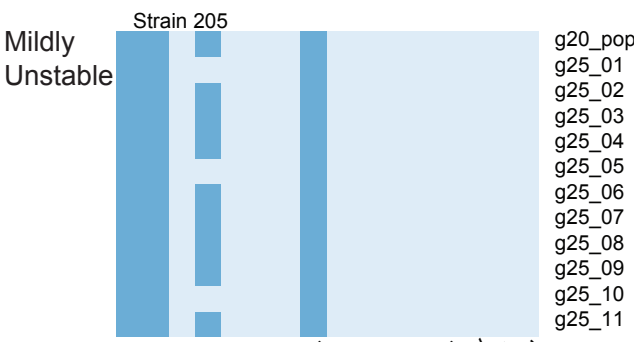

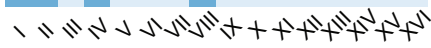

Strain 207

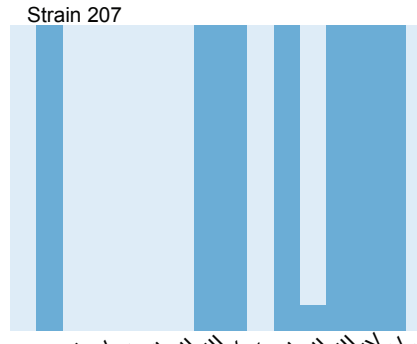

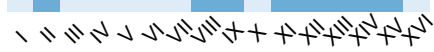

\section{Strain 214}

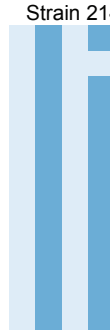

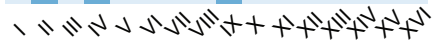

Strain 223
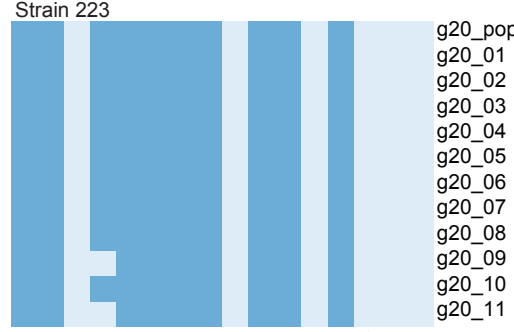

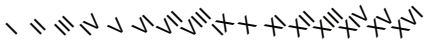

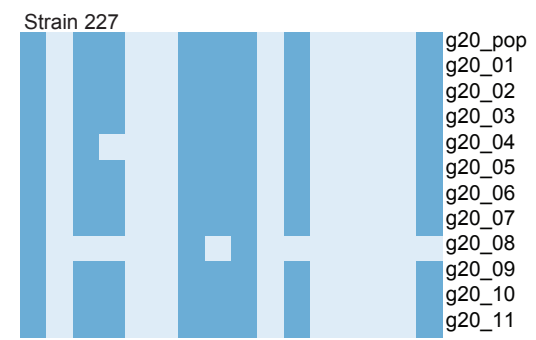

g20_pop g25_01 g25_02 g25 03

g25_04

g25_05

g25_06

g25_07

g25 08

g25_09

g25_10

g25_11

g20_pop g20_01 g20_02 g20 03 g20_04 g20_05 g20_06 g20_07 g20_08 g20_09 g20 10 g20_11

g20_pop g20_01 g20_02 2003 g20_04 g20_06 g20_07 2008 g20 10 g20_11

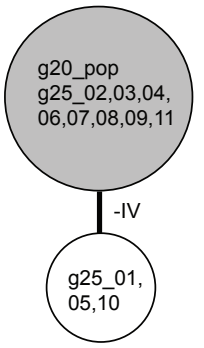

1 CIN event Mildly Unstable

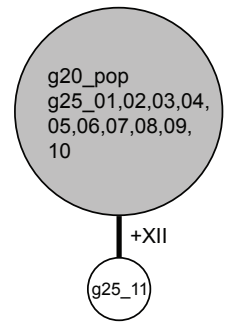

$1 \mathrm{CIN}$ event Mildly Unstable

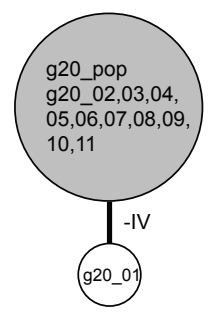

$1 \mathrm{CIN}$ event Mildly Unstable

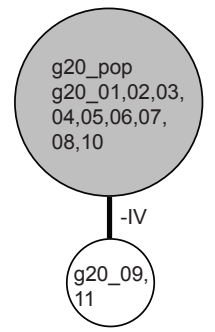

$1 \mathrm{CIN}$ event Mildly Unstable

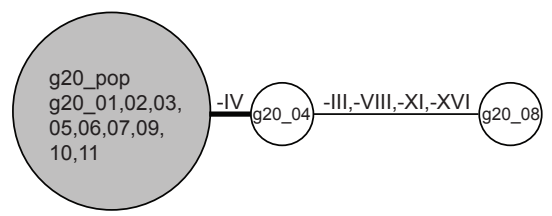

$1 \mathrm{CIN}$ event Mildly Unstable 


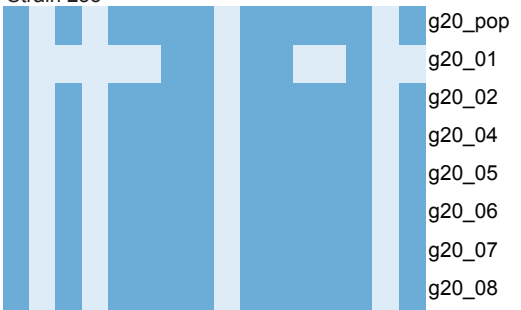

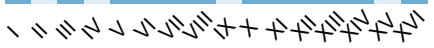
Strain 236

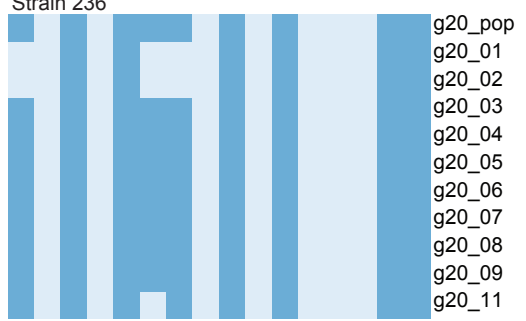

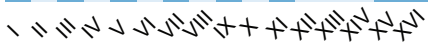

Strain 239
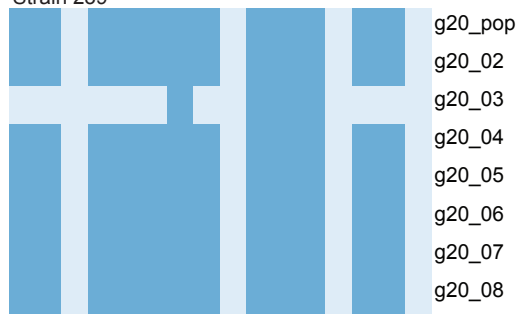

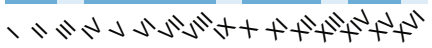

Strain 247
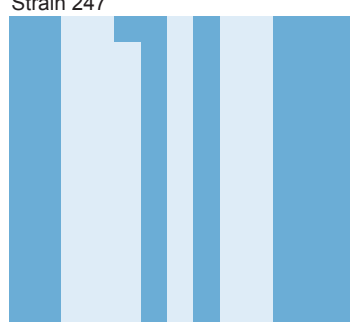
g20_pop g25_01
g25_02
g25_03
g25_04
g25_05
g25_06
g25_07
g25_08
g25_09
g25 10
g25_11

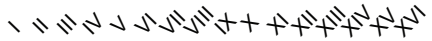

Strain 215

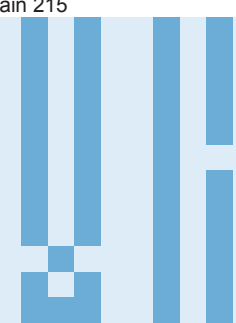

g20_pop g20_01

g20_02

g20_03

g20_04

g20_05

g20_06

g20_07

g20_08

g20_09

g20 10

g20_11

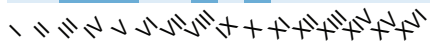

Strain 228

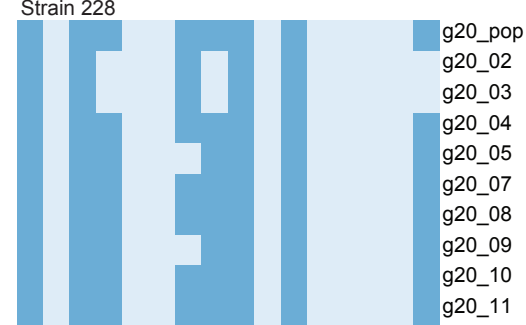

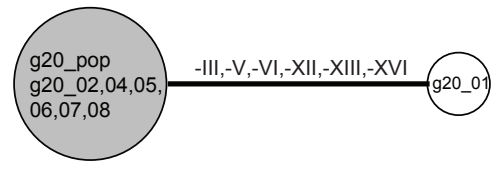

1 CIN event

Mildly Unstable

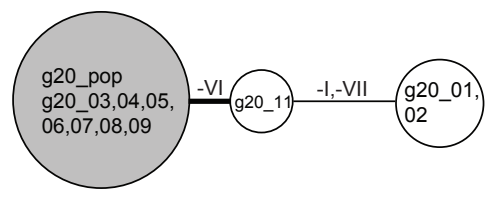

1 CIN event

Mildly Unstable

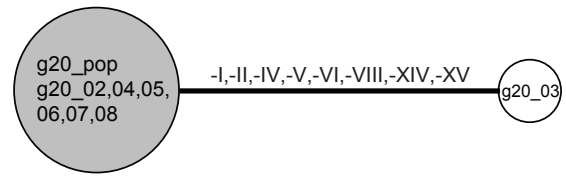

1 CIN event Mildly Unstable

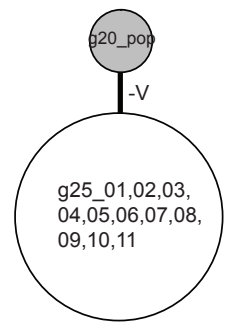

$1 \mathrm{CIN}$ event

Mildly Unstable

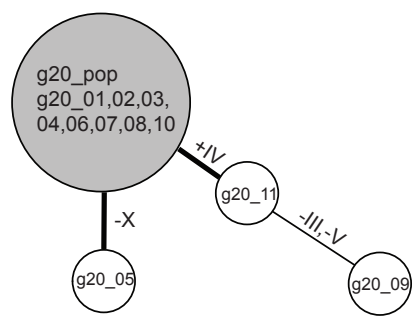

2 CIN events

Highly Unstable

2 CIN events

Highly Unstable

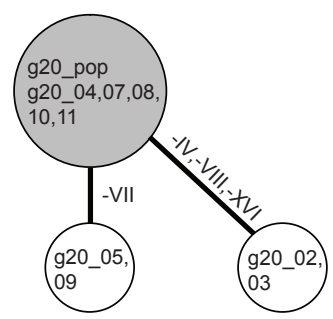

Figure S7 (Page 3) 


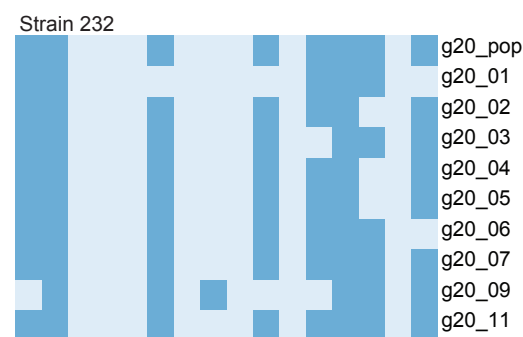

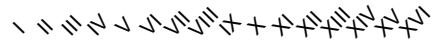

Strain 240
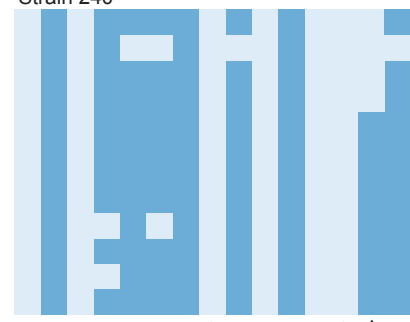

g20_pop g20_01 g20_02

g20_03 g20_04

g20 05

g20_06

g20_07

g20 08

g20_09

g20_10

g20_11

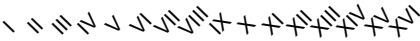

Strain 241
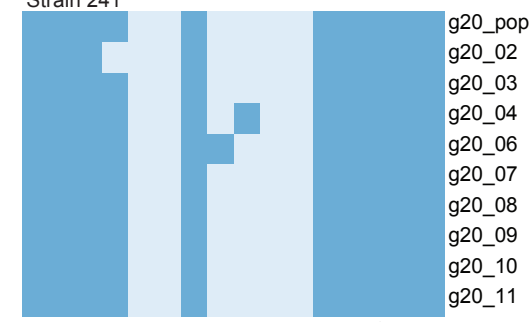

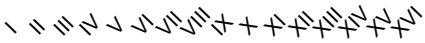

Strain 243

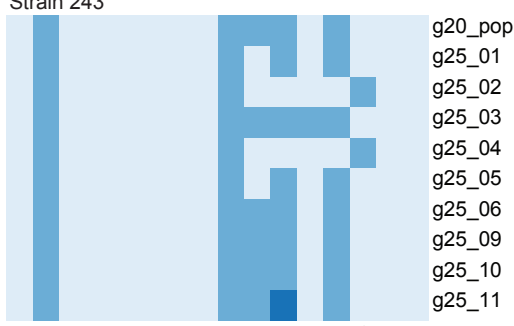

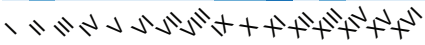

Strain 251
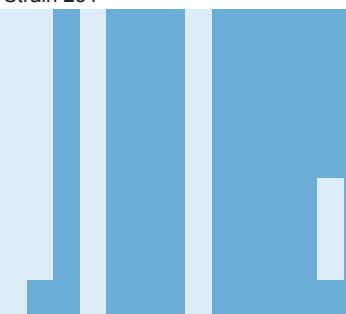

g20_pop

g20_01

g20_02

g20_04

g20_05

g20_06

g20_07

g20_08

g20_09

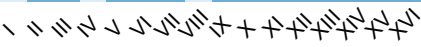

Strain 252

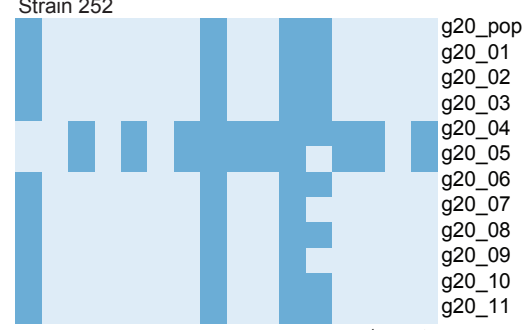

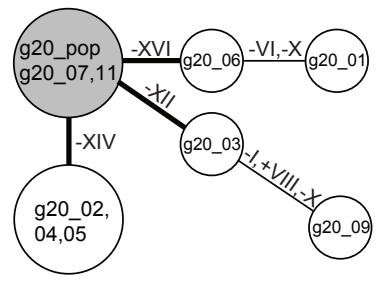

3 CIN events Highly Unstable

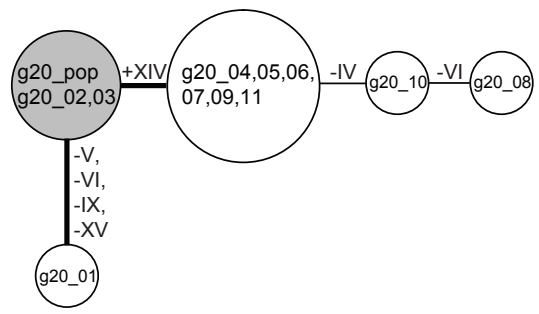

2 CIN events Highly Unstable

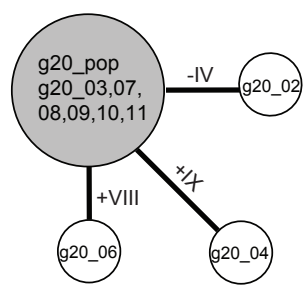

3 CIN events Highly Unstable

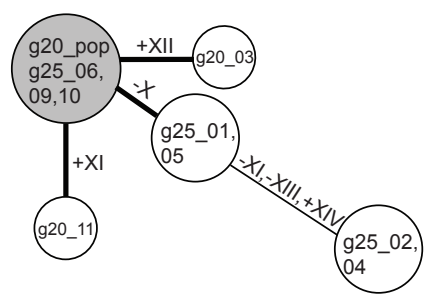

3 CIN events Highly Unstable

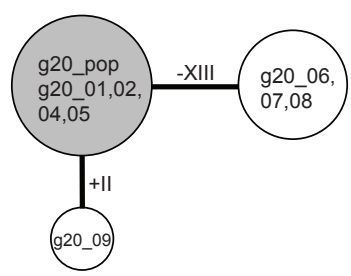

2 CIN events Highly Unstable

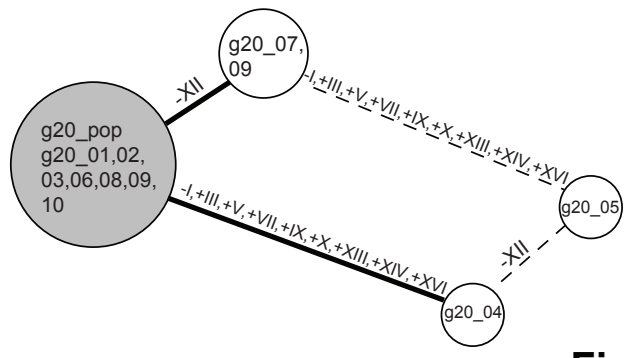

2 CIN events

Highly Unstable

Figure S7 (Page 4) 